\title{
Design and Development of a Learning Design Virtual Internship Program
}

\author{
Dana Ruggiero and Jeff Boehm \\ Bath Spa University
}

\begin{abstract}
Incorporation of practical experience in learning design and technology education has long been accepted as an important step in the developmental process of future learning designers. The proliferation of adult online education has increased the number of graduate students who are in need of a practical internship placement but have limited options due to work and family obligations. An international virtual internship program provides opportunity for non-traditional students to participate in a practical experience regardless of their physical location and other obstacles.
\end{abstract}

Keywords: graduate program, learning design, internship, development, virtual environment

\section{Introduction}

Students in learning design and technology related disciplines not only learn theory, but also learn through real-world experiential learning. The digital learning landscape is a global one, characterized by pockets of innovative activity in unexpected places. These two factors indicate that (a) an internship component is essential if learning design programs are to be successful in providing students with up-to-date and relevant digital learning knowledge and skills, and (b) in order to provide our students with exposure to a global environment, we should endeavour to provide internship opportunities that are not limited by location. A possible approach to meeting these two needs is to create international virtual internship programs. This paper explores the design and subsequent implementation of an international virtual internship program for novice learning designers. We begin with a discussion of the need for internships as a bridge between theory and practice, followed by a review of the literature relating to the emerging concept of virtual internships. The paper continues with a description of the design and development of the Bath Spa University Virtual Internship Program (VIP). We conclude with lessons learned and suggestions for future iterations of the internship program.

\section{Bridging Theory and Practice}

Learning Design (LD) is a complex, ill-structured, problem solving activity that involves decisionmaking procedures (Jonassen, 1997; Simon, 1977). The learning design process is dependent upon the decisions that learning designers make (Kozma, 1994; Rowland, 1993). Decisions made by learning 
designers vary significantly between novices and experts. Furthermore, research has indicated that expertise in other domains does not equal good decision-making in learning design (Clark \& Mayer, 2011; Crismond, 2001; Rowland, 1992). Novices (in this case graduate students), may have an appropriate knowledge base, but often lack the teaching and design experience necessary for efficient and effective problem solving (Ertmer et al., 2008; Ertmer, York \& Gedik, 2009). Perez \& Emery (1995) purport that a designer must have ten years of consistent hands-on experience in order to obtain the necessary expertise. One way to ensure that novice learning designers are graduating with at least some field experience is to require them to have practical intern placements within the education or industry sectors.

Research indicates that field experiences are an important component of a professional graduate program and also serve to build connections between students and future employers (Bohling, Hough, Krinsky, Saleem, \& Stevens, 2012; Weidman, Twale, \& Stein, 2001). Internships tend to be site-based, especially in learning design programs. To participate, students need to be located near an approved internship site. This arrangement presents non-traditional students, working students, and rural students with several unsatisfactory options: to temporarily relocate, to travel long distances, to take time off from their current jobs, or to forgo participating in an internship experience altogether. These options are usually not possible or create hardship for the student.

\section{Moving from Face-to-Face to Online Environments}

Advances in technology, including high-speed Internet connections and low-cost portable devices, have made the virtual workspace a reality. Recent research (Ruth \& Chaudhry, 2008; Shin, El Sawy, Sheng, \& Higa, 2000; Weinert, Maier, Laumer \& Weitzel, 2014) has revealed the following benefits of telework (remote or virtual work): an increase in employee productivity, an increase in employee satisfaction, and a reduction in employer costs. According to a recent study, three out of every four companies surveyed employ some virtual workers, and it is expected that the numbers of virtual workers employed in those companies is expected to double every year over the next decade (Mulki, Bardhi, Lassk, \& Nanavaty-Dahl, 2012). Of the U.S. employee workforce, 2.6\% (3.3 million people) consider home their primary place of work. With virtual work growing nearly $80 \%$ from 2005 (Global Workplace Analytics, 2013), the virtual workforce is expected to continue to grow.

Benefits of virtual internships. Higher education institutions are beginning to recognize the value of virtual internships as valid experiential learning opportunities for acquiring professional skills and competencies. Columbia University's Virtual Internship Program website provides this compelling rationale: "Currently, over 8 million people work virtually across the United States and increasingly, companies and organizations are exploring the virtual workplace” (Columbia VIP Program, 2014).

Traditional internship experiences benefit students by reinforcing their academic learning (Kuh, 1993), increasing their self-confidence (Callanan \& Benzing, 2004), and providing an opportunity to establish professional contacts (Mayernik et al., 2015). However, traditional internship experiences also pose challenges such as those mentioned previously. Virtual internships offer the same benefits as traditional internships, but also reduce the need for relocation or unnecessary travel while giving the intern an opportunity to gain experience working in a virtual environment. In fact, virtual internships add particular value to traditional education, as well as to distance education (Jeske \& Axtell, 2014). 
How do virtual internships promote learning of LD content/concepts? Virtual internships, particularly at the graduate level, provide a number of unique affordances that have been demonstrated to increase student learning (Conroy \& Khan, 2009; Jeske \& Axtell, 2014, 2016). The most prominent of these affordances include (a) engagement with real-world designs, (b) interaction with diverse perspectives, and (c) actual participation in professional realities. Specifically, in learning design, these affordances provide learners with an opportunity to simultaneously learn LD content/concepts, as well as develop professional soft skills. However, best practices in design and development of such programs have yet to be established. A greater understanding of how learning theory and applications of learning design can be used to design and develop virtual internships is needed.

Linking learning theory and virtual internships. The current literature on online distance learning provides some insight into the value of virtual learning. The design of a virtual internship draws upon research within the constructivist learning paradigm (Garrido-Inigo \& Rodriguez-Moreno, 2013), virtual internships being by nature constructivist activities (Wang, 2009). Constructivists believe that learning is a process of interpreting, building, and modifying our understanding of reality based upon life's experiences (Jonassen, 1997; Shea \& Bidjerano, 2013). Dewey (1966) believed that knowledge emerges only from situations in which learners have to draw them out of meaningful experiences. Virtual internships provide the opportunity for students to apply their subject matter expertise and theoretical background to the workplace in order to create new knowledge and skills through social negotiation with both the learning technology mentor and the academic client. Over the past twenty years the field of online learning has made multiple contributions to the theory of distance learning in relation to constructivism, one of which is the Community of Inquiry framework (CoI) (Garrison, Anderson \& Archer, 2010). The constructivist nature of the CoI framework links the ideas of presence (teaching, social and cognitive) to the dynamics of design in online learning. To consider the affordances and challenges present in online distance learning, many scholars have used the CoI framework to ensure that research examinations are completed in an efficient and meaningful way (Garrison, Anderson, \& Archer 2010). Comprised of three constructs-social, teaching, and cognitive presence-the CoI framework contends that the educational experience for students is determined by how these three elements interrelate. Student reports of isolation relate to the social construct while teaching presence is linked to how instructors approach specific challenges in the virtual classroom. One of the key issues in online distance learning has been how to engage students meaningfully while maintaining a scalable system for instruction. Good practices in learning design have only taken this so far and current research inquiries are examining how emerging technologies can impact online distance learning (Reiser \& Dempsey, 2011). In 2011, Reiser and Dempsey argued that piecemeal change in the field of learning design and technology was inadequate for meeting student and community needs in the information age. Learning theory in isolation is not enough. Learning and performance typically needs to occur in the context of organizations such as school districts, universities, businesses, and government institutions. The individual's technological skill alone is not sufficient; they must work in concert with others in a structured environment (Rienties, Tempelaar, Giesbers, Segers, \& Gijselaers, 2014). The opportunity to offer virtual internships using computer-mediated communication is particularly promising when the internship placement is valued as an academic class worthy of pedagogical improvement (Black \& Bachman, 2007).

Both the constructivist paradigm and the CoI framework lend credence to the value of virtual internships to bridge the gap between the digital learning classroom and the workplace. The focus of the Bath Spa University Virtual Internship Program, which includes partnerships between U.S. 
students and our site in the United Kingdom, is learning design. This focus highlights one of the major benefits of a virtual internship built upon a constructivist paradigm and the CoI framework: the ability for students to learn from experienced professionals and to become part of a community of learners, regardless of geographic location.

Rationale for digital learning development. There is a rapidly increasing demand for learning design professionals who can manage the training and development needs generated by a burgeoning online and blended education movement in colleges, universities, industry, and the government (E-Learning Market Trends \& Forecasting, 2014). This relatively new and pressing need has created a rising demand for learning designers, learning technologists (LTs), and curriculum creators trained to apply the latest tools and methods for effective learning design. They must be able to effectively create and manage material that is either originally digital or converted to digital form for the purposes of learning and training.

In the VIP program, learning designers leverage learning technologies to assist faculty in evolving real-world, face-to-face courses to include blended teaching and learning methodology. They create fully or mostly online courses and have strong theoretical backgrounds in how people learn while partnering with teaching staff and a mentor to leverage that knowledge and experience for effective course design. According to the Occupational Outlook Handbook (2011), employment of learning designers is expected to increase up to $14 \%$ by 2022. In response to this growing demand, master's programs that have been traditionally taught face-to-face have expanded to include online only options. New courses are being developed that build on competency based assessment (Chang, 2006) of which field experience is an important component, allowing students to apply what they have learned, while building beneficial connections with potential future employers.

\section{Learning Design in the United Kingdom and United States}

The field of Learning Design is also known as Learning Design and Technology or Instructional Design and Technology in the United States. In the UK, the term "e-learning technology" is sometimes used as well as "digital education." A search of the postgraduate school search site, www.postgraduatesearch.com ${ }^{1}$, for any of the above designations yields only one university with a program dedicated to "e-learning technology," another with a MSc in Digital Education, and only one university offering an MA in Virtual Worlds. However, a search for Learning and Technology yields a list of 16 universities (including the three already mentioned). We believe that the lack of parity between what United States and UK universities call "learning design" has impacted the opportunities for international internships. Unlike business or engineering, learning design draws from the cultural context in which it is nested; in this case there is a mismatch between the common education practices in the United States and UK that had to be addressed.

The uncommonalities of the common language. One benefit of an international experience is that interns are introduced to the different ways in which other cultures communicate and operate. Even though most people would probably say that there are few cultural differences between the US and the UK, the author's experiences prove otherwise. For instance, in the UK, modules are what the US (and Blackboard) calls classes, and a class in the UK refers to a student cohort (e.g., Class of 2014). There are other obstacles to communication as well. Cross-Atlantic timing

\footnotetext{
${ }^{1}$ The term, 'postgraduate' in the UK means after the undergraduate degree.
} 
can create problems when setting up meetings: there is a five hour time difference between GMT and the Eastern Time Zone of the US. If interns are in California, it is an eight hour difference. The school calendar in the UK runs from October into July. In the US, it runs from September into June. Differences in grading systems ("marking" in the UK) can also cause problems for interns creating rubrics and assessment structures. Although the UK uses formative assessments, they are generally not marked. Indeed, there is very little summative assessment. Rarely are reading knowledge or basic terminologies assessed as they would be in the US. Attendance, although considered essential in the UK, is not factored into a typical module assessment scheme. In the US attendance is often counted toward the final grade as participation. All of these subtle differences mean that interns from a country such as the US need to learn to adapt to a new set of meanings within a different cultural environment.

The perfect storm. Bath Spa University (BSU) is rapidly growing its international and nontraditional student base, while also encouraging staff to incorporate blended learning activities into traditional on-site classrooms. The growing international and non-traditional student base requires that non-traditional learning environments be put in place. Because the university is one of the smaller universities in the United Kingdom, there are relatively few staff dedicated to developing online and blended learning materials. The combination of the two situations, BSU's growing nontraditional student base and other universities' needs for intern placements, creates a perfect storm for the development of an international virtual internship program.

So, in order to overcome these obstacles and to provide their academic staff an opportunity to work with international learning design graduates, the Learning Technology team at Bath Spa University developed a virtual internship program. The Virtual Internship Program (VIP) is modelled after existing field-based professional experiences that take place on-site, such as at a higher education institution. Consistent with partnership university internship and practicum requirements, students are required to work up to 90 hours for a maximum of three graduate level credits. Bath Spa provides a learning technology mentor to supervise the structured learning experience and acts as the host organization providing the academic client and design task. The differentiating factor between the VIP and existing learning design internship opportunities is that all the work is performed virtually through the university's virtual learning environment. Also, unlike site-based internships, virtual internship hours are flexible, making them more convenient for the interns.

\section{Design and Pilot Project}

When the university hired five learning technologists in 2012, it was in part to forward an impetus towards online distance learning. In addition to serving as campus leaders in learning technologies, each of the LT's would also be assigned to at least a 30\% teaching load. With the online remit, 30\% teaching, and a teaching staff of over 500 having varying degrees of technological competencies spread across four campuses, it quickly became apparent that the team would need help with the hands-on portion of developing these online initiatives. The issues were compounded as the LTs learned that several of the teaching staff were not skilled in even basic uses of our virtual learning environment, such as uploading files and attaching rubrics to assignments. So with these complications in mind, we moved forward with the design phase of our program.

Design. The first step in designing the virtual internship program was to decide on the learning outcomes and goals for the program. In the spring of 2013 our team of learning technologists 
began the process by researching other programs. We found that no other institution of higher education hosted a virtual internship programs for graduate students in learning design. With no other virtual intern programs to benchmark against, we had both the freedom to experiment and the challenge of starting a program with no reference points. We chose to base our framework on the CoI model (Garrison, Anderson, \& Archer, 2010) and the Association of Educational Communications and Technology Code of Professional Ethics (2007), which focuses on commitment to the self, society, and profession. From the code of ethics we proposed three goals for the program:

- To provide opportunities for graduate students to participate in a structured field experience, regardless of where they live.

- To pair BSU staff with competent learning designers, making it possible for the staff to effectively implement learning design in their own modules.

- To develop an innovative model for virtual internships that can be replicated for learning design students around the world.

Using the goals of the program as a framework, and the advice gathered from surveys of BSU staff and learning design program advisors in the United States, we developed the following learning outcomes:

- The virtual intern will hone professional skills that include timely communication, meeting deadlines, and working with clients by participating in a real-world environment in higher education.

- Working in conjunction with both a client and a mentor and using the Blackboard Virtual Learning Environment, virtual interns will build a real-world learning module constructed for either a blended or online learning environment.

- The interns will create a virtual tour of their learning module rebuild, explaining the application of learning and design theories that they used.

- The virtual intern will reflect upon their experiences in an online journal.

In addition to working with their client, all students were expected to meet the following module requirements:

- Participation in weekly discussions in an online learning management system.

- Communication about their internship experience through weekly journal posts.

- Attendance at a minimum of two of the three web conferences during the term.

- Completion of an online evaluation of the internship.

- Submission of a storyboard, usability test, and final video showcasing their redesigned module.

Pilot. After an expert review of the proposed learning design internship we ran a pilot in the winter of 2013 with two students from graduate programs in the United States. Both students were female and in the last terms of their graduate degrees. Each intern was paired with a BSU learning technologist (serving as a mentor) and a client. In order to help increase knowledge of best practices across the university, we decided that the mentor should not be based in the same department as the client. The pilot program lasted 10 weeks and each of the interns met with their client and learning technologist mentor at least weekly to create online and blended modules from existing face to face modules. From this first cohort we learned four important lessons: 
- A student space was necessary on the learning management system for the interns to communicate with each other.

- Learning technologists and clients would have to come from the same department, as there were too many differences between departments for LTs to be able to adequately advise on academic detail.

- The length of the internship was appropriate but the assignments needed to be spread more evenly across the duration of the internship.

- The client needed to have all of the needed module content material prepared prior to the start of the program.

We decided we would offer the internship two times a year with varying timelines. The summer program would be 8 weeks and our largest cohort $(n=10-19)$; the autumn program would be 12 weeks and medium sized $(n=7-9)$. These decisions were made based on university logistics: staff are busiest in the spring with dissertation marking, and more apt to be going on break in the summer, while the autumn is the start of the term and staff are more likely to be unable to pull together their material in time. We then launched the VIP in the summer of 2014.

\section{Implementation}

First cohort: Summer 2014. One of the first steps in creating a cohort of virtual interns is to create an online portal. We created a module called, 'June 14 Interns' in Blackboard and populated it with weekly discussion questions, an intern journal, video updates, and an assignment Dropbox (See Figure 1). Second, we paired the incoming interns with their learning technology mentors and clients. Clients were chosen from the staff of four schools: Art and Design, Education, Music, and Science and Enterprise. 


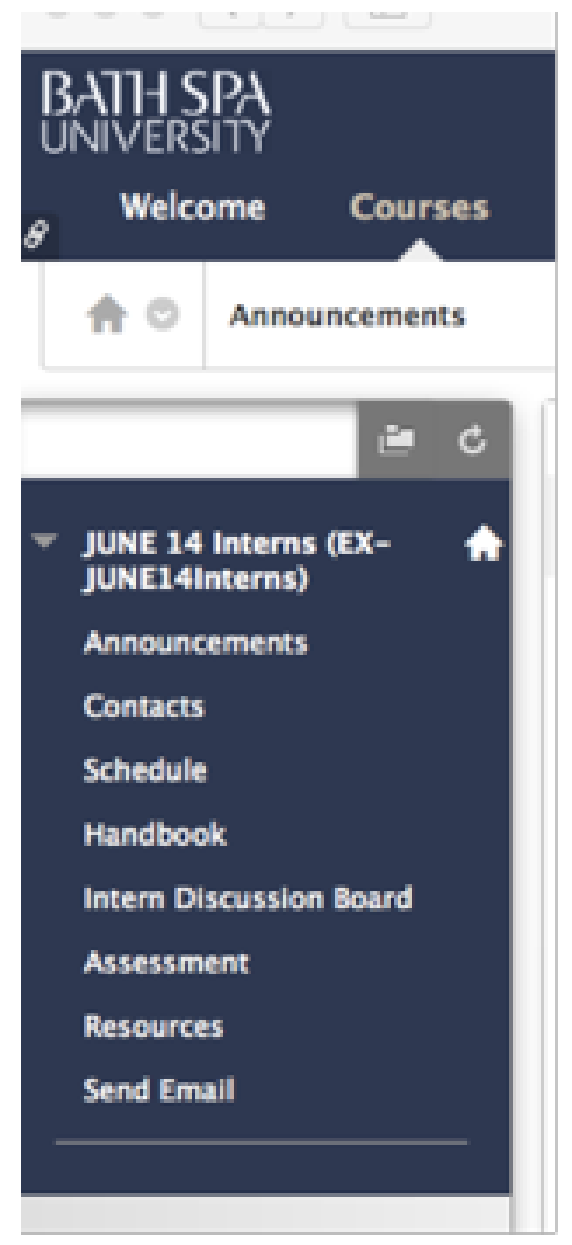

Figure 1. June 14 intern module sidebar.

The internship was divided into three key sections:

1. Introduction and Storyboarding - From week 1 to week 3 the interns spent their time familiarizing themselves with our learning management system, Blackboard. They also met with their learning technology mentor three times and their client twice. At the end of week 1 the intern had done a review of the current module and set up a client meeting for the following week to discuss their client's goals for the module. In week 2 and 3 they developed a comprehensive storyboard of their redesign and met with the learning technology mentor to create a cohesive development plan. At the end of week 3 , the intern presented the redesign to their client and made adjustments accordingly.

2. Building - From week 4 to week 6 the interns spent their time building the redesigned modules, creating learning objects, and using learning theory to create a progression of content and reflection for the modules. They also met with their learning technology mentor three times and their client once. At the end of week 4 the intern had created a detailed template for the new module. In week 5 and 6 they then populated that template with the learning objects and instruction to build the module. During this time interns also worked with their client to produce short lecture videos or audio tracks for the module.

3. Usability and Presentation - Week 7 and week 8 were used for conducting usability tests and creating a final presentation video for the client. The usability test required the interns to 
find three to five people in the target audience (i.e., graduate students) to provide feedback regarding the functionality of the module. After the usability testing was complete the interns used the feedback to make any necessary changes to the design or navigation of the module. The interns then created a five-minute walkthrough video that showed the client the steps they had taken to redesign their content from a face-to-face module to an online or blended experience. At the end of week 8 the interns met a final time with the client to deliver the final product and also met with their learning technology mentor to complete a post-assessment on the entire experience.

Three key points arose from this iteration of the internship:

- Interns did better with acclimating to the pace when we held large group meetings for the key assignments.

- Usability testing had to be scaled down to accommodate the timescale of the internship.

- Client interaction would be required at least three times, but should the intern and client decide to meet more often they could choose to do so.

Lessons learned from Cohort 1. Intern feedback from the first cohort was collected weekly during mentor meetings and also at the final post-assessment meeting. Large group meetings via video conferencing were successful so we decided to hold more of them for the following cohort. Our usability testing requirement proved to be somewhat problematic. We asked interns to test their redesign with age-appropriate subjects (determined by the module's target audience). Because this internship took place during the summer and the interns were working remotely (not near a campus), it was difficult to find testing candidates. We therefore reduced the required number of usability tests to two with someone at either the undergraduate or graduate level. Some interns and clients were meeting twice weekly to talk about the redesign, but some interns and clients assumed that they were limited to three meetings. To rectify this misunderstanding we sent out an email stating that three meetings was the minimum but that they could meet as many times as necessary. The process of the internship also revealed that not all clients were able to provide as clear of a remit at the beginning, so we created a first-time interview structure to be implemented in the following cohort. Using the intern feedback we adopted the changes and prepared for the next cohort.

Second cohort: Autumn 2014. We launched the second cohort in September 2014 with 9 interns across three schools: Education; Music and Performing Arts; and Society, Enterprise, and Engagement. This cohort consisted of mainly distance learning students from four major universities in the United States and ended in December 2014. Because this cohort ran during the academic term, the time-span was four weeks longer than the summer cohort. The structure of the program was adapted to work within the timeframe.

1. Introduction - From week 1 to week 3 interns spent their time familiarizing themselves with the internship, the university, the client, and the framework of the learning management system. During this time they met as a large group for the introduction of the program and weekly with their learning technology mentor. At the end of the three weeks they met with their client to discuss the project and ask questions about the content. For this cohort we provided the interns with an interview protocol that ensured they asked their client questions about teaching style, experience in teaching online, and expectations. 
2. Storyboarding - Week 4 and week 5 were spent consulting with the learning technology mentor as the intern constructed a storyboard for the redesign. To create agreement between what the client wanted and what the intern was producing the storyboarding process was dictated in some respects. For example, storyboards had to include a visual framework for all of the redesigned components at each level rather than only a basic overview of the module. At the end of week 5 the intern was required to meet with their client to present the storyboard and get approval for the redesign.

3. Building - Interns built in their sandbox modules from week 6 to week 10. During this time they were required to report on their progress during the weekly mentor meetings and went through iterations of the design to ensure a consistent delivery across the sections of the new module. In addition to building, the interns took part in the discussion forum where they shared best practices, new web 2.0 tools, and tips on how to shortcut some of the more bulky tasks such as repeatedly recreating icons.

4. Usability and Presentation - To ensure that the usability tests followed a specific protocol designed to simulate actual user experience, interns were required to turn in their usability plans by the end of week 10. During week 11 the interns conducted usability testing in pairs and peer reviewed each other's protocols for professional development and consistency. After usability testing, the interns produced a five-minute video that showcased their design process and uploaded this to the intern discussion board to share.

Lessons learned from Cohort 2. At the end of this cohort we had implemented new protocols to control the quality of the redesigned modules, such as: peer review of the redesign, a longer storyboarding period, and the sharing of final presentations. By making the interns accountable to their peers in addition to their clients we had a more consistent style of redesign across the program. Intern feedback was positive for these changes and the extended timeframe allowed the clients to form closer professional relationships with their interns. Additionally, the interns were able to create in iterations rather than in a single design stage, and then share that process in our large group meetings. At the end of the second cohort we had learned two key lessons:

- We needed a protocol for when an intern needed to leave the program.

- Interns were participating in this program for credit as well as experience and we needed a way to give access to the final modules to the partnering universities.

In order to address the first issue for the next cohort we had to review the process by which an intern could drop out of the program. During the second cohort one of our interns understandably had to drop out due to a death in the family. However, we had an intern in the first cohort and one in the second who just stopped communicating with their mentors and clients. After two weeks we contacted the partnering university to inform their advisors of non-participation and had their access to our services cut off. To protect the program and the intern experience we decided to address how interns should communicate their leaving the program in the large group meeting for the next cohort. All of our interns were participating in this program for credit in their graduate degrees and some of the universities required access to the final redesign. During the first and second cohort individual mentors had given access to the universities through guest access but that did not allow our university to track who was accessing the system. To solve this problem we assigned secure user IDs to each of the universities and then enrolled these university users on the their students' modules. 
Third cohort: Summer 2015. For the third cohort we focused on cementing our processes and procedures while maintaining a high level of communication and interaction among mentors, clients, and interns. This cohort lasted eight weeks from the beginning of June and consisted of twelve interns from around the world, representing seven universities. While we know that a ten or twelve week internship allows more connections to form and a more detailed product to emerge, calendar constraints make it so there is not enough time to conduct an internship longer than eight weeks in the summer. In fact, the learning technology team has a difficult time recruiting clients for this timeframe as academics are usually on vacation after mid-July.

This cohort followed the same basic structure as the first cohort with the first three weeks consisting of introduction to the program and storyboarding, weeks 4-6 used as building weeks, week 7 for usability tests and week 8 for the final presentation. However, this cohort was supported more fully with the integration of the lessons learned from the prior two cohorts and there was a more detailed structure in place for each of these activities. In addition, the interns were allowed to meet freely with their clients and create a schedule that worked for the individual pairings. Given the constrained timeframe we also introduced a special project for a trio of interns to create a fully online international research module for graduate students. The presence of a special project worked so well that this opportunity will become a permanent feature of the virtual internship program. One key component of this cohort was the development of procedures developed by the interns for certain segments of the internship. For example, the interns created a strategy for incorporating internal links within the module design and icon design for a more cohesive look across all projects. To accomplish this the learning technology team asked the interns what would make the program more applicable to their degree and these were the suggestions that were returned. In addition, we introduced a more defined journaling system for the interns where they had to use their weekly entries to reflect on their design process using specific learning theories. At the end of this cohort we had learned one very important lesson: design is as fluid as the academic who is teaching the module and as adaptable as the intern redesigning the module.

Lessons Learned from Cohort 3. While the learning technology team wanted to ensure a consistent experience across the program, we realized late in this cohort that it is not possible to control the relationship that forms between the client and intern. Some pairings were successful because of the structure of the program, but many more were successful in spite of it so the mentors worked with the interns to meet the need of the client on an individual basis. One of the key moments in this cohort was the realization that we have hit capacity for the program if we are to promote bespoke experiences rather than ensuring a consistent experience where everyone gets the same basic experience. For future cohorts, the learning technology team plans on implementing a short training for the clients to increase their awareness of the intern experience and how they can be positive clients through honest feedback and clear expectations.

\section{Conclusion and Discussion}

In this section we will review the program goals, address the sustainability and growth of this program to pair with our MSc Learning and Innovation degree, and examine cultural issues in educational systems. We conclude with an introduction to our virtual attachment theory (VAT) that reimagines Ainsworth and Bowlbys' attachment theory (1991) in terms of distance learning and adult mentoring. We have managed to provide opportunities for graduate students to participate in a structured field experience, regardless of where they live. Interns have lived in places from both coasts and the 
Midwest of the US, as well as Japan. By being flexible with the communication tools that we use, and training the interns to utilise the Google education suite, we have been able to mitigate issues with scheduling meetings and other potential communication problems. The use of modern communication technologies and flexible scheduling has put everybody on an even footing.

The number of BSU staff working with learning designers has expanded as word of our successes has spread. The only two limitations at this point is the availability of the LTs for mentoring and staff being able to bring prepared materials into the internship.

We have developed an innovative model for virtual internships, which can be replicated for LD internships that focus on other topics. Built on the CoI framework, this program helps inform a community of practice that will hopefully follow the interns throughout their careers. Continuing inquiries from other institutions regarding this program verify that is fills a need in the learning design community.

Currently, the program is sustainable because each LT will only accept the number of interns that we know we can handle during a given period. It is our hope that as the program grows, our connections with other programs and past interns will develop into new partnerships. Although there is currently sufficient demand for converting modules from face-to-face to blended and online learning environments, we do not know how much demand there will be in the future. We also have questions as to how this program will continue as we develop our own MSc in Learning and Innovation degree. Will our own students take up all the availability of spots, or might we be able to establish an exchange relationship with other institutions?

We addressed the cultural issues such as terminology and grading differences by providing the interns with an introductory video and chart that shows the comparisons and contrasts in our systems. We also address these issues as they arise during our one-to-one mentor/intern meetings, and interns have the option to post questions in the discussion forum. Finally, as we progressed through the design, development and implementation of this virtual internship program we began to notice themes that helped us build the virtual attachment theory.

The Virtual Attachment Theory (VAT). Ainsworth and Bowlby (1991) expanded the attachment theory, where a figure serves as a secure base from which an infant can explore the world, to an empirically demonstrated concept that has guided developmental psychology for the past thirty years. We propose that when faced with a new situation, adults will seek a secure base from which to explore. In a virtual environment that security will come from the mentoring relationship that has been developed within the CoI framework. The VAT has been applied to the last three cohorts of virtual interns and we have analysed the patterns of communication, work quality, and relationships between the virtual intern/client, virtual intern/mentor, and mentor/client. VAT follows the adult attachment theory while considering the unique principles of presence presented by the CoI framework. The key principles of the VAT are communication, guidance, and work quality.

Learners with a secure attachment to their mentor who have a secure connection with their clients demonstrate independence, a clear and consistent communication style, and a willingness to be guided by the experience of the learning technology mentor. Learners with a preoccupied attachment to their mentor who have mentors with a secure, preoccupied, or dismissing connection with their clients demonstrate an inconsistency in work quality and communication. Finally, learners with a 
dismissing attachment to their mentors who have mentors with a secure or preoccupied connection with their clients often do not maintain contact, make excuses early on in the project and ultimately may not complete the project or drop out of the program entirely. In practical terms this suggests that the level of collegiality among mentors and clients affects the relationship that the intern and client develop. Explicit, clear communication between clients, mentors, and interns during the virtual internship led to secure attachments and internships that ended in completed projects meeting all of the criteria.

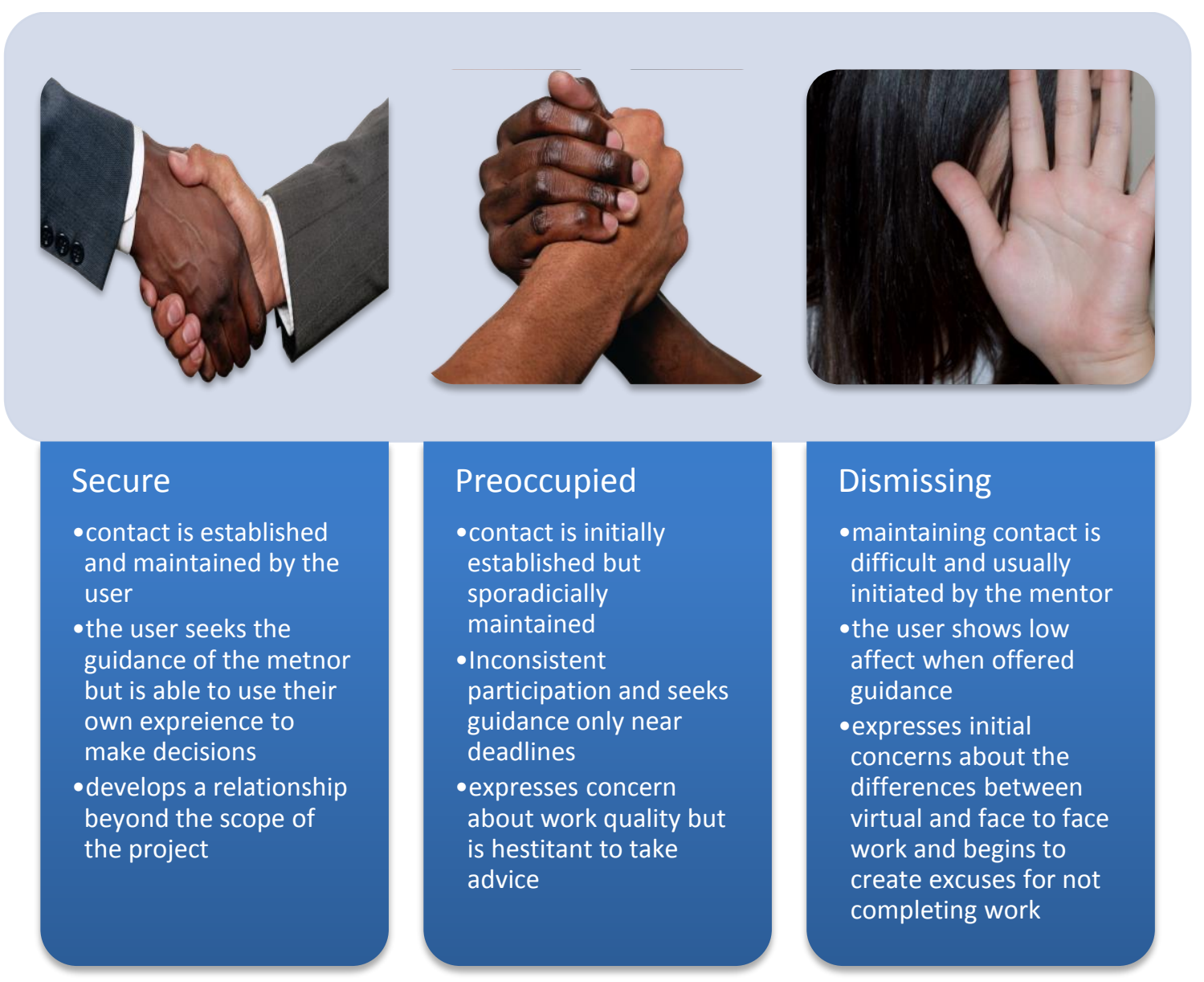

Figure 2. Understanding Virtual Attachment Theory.

Applying attachment theory to the virtual internship program has informed the design and development of the subsequent cohorts of virtual internships. For example, at first we only considered the intern's background in terms of courses completed and we expanded that to questioning their university advisors about their communication style and relationship with students and professors. Now, on completing our fifth cohort, we have concluded that the initial connection that the mentor and learner make is crucial to the expectations of the learner and sets the tone for the remainder of the relationship. The limitations of this study are integrally tied to its sustainability. Small numbers, long stretches between internships, choosing interns according to a set of criteria, and selecting clients from a pool of volunteer lecturers are all variables that limit this study. This also has limited development of the VAT and empirically testing its features among a larger cohort of virtual interns. 
Future research is needed to further test the VAT and the impact of the framework on the relationship and work quality of program development.

\section{References}

Association of Educational Communication and Technology Code of Professional Ethics. (2007). Retrieved from http://aect.siteym.com/members/group content view.asp?group=91131\&id=309963

Ainsworth, M. S., \& Bowlby, J. (1991). An ethological approach to personality development. American Psychologist, 46(4), 333.

Black, G., \& Bachman, V. (2007). Virtual internships in the business accounting curriculum: A feasibility study. Journal of Business Administration Online, 6(2), 1-15.

Boling, E.C., Hough, M., Krinsky, H., Saleem, H., \& Stevens, M. (2012). Cutting the distance in distance education: Perspectives on what promotes positive, online learning experiences. The Internet and Higher Education, 15(2), 118-126.

Callanan, G., \& Benzing, C. (2004). Assessing the role of internships in the career-oriented employment of graduating college students. Education+ Training, 46(2), 82-89.

Chang, C.C. (2006). Development of competency-based web learning material and effect evaluation of self-directed learning aptitudes on learning achievements. Interactive Learning Environments, 14(3), 265-286.

Clark, R.C., \& Mayer, R.E. (2011). E-learning and the science of instruction: Proven guidelines for consumers and designers of multimedia learning ( $3^{\text {rd }}$ ed.). San Francisco, CA: Pfeiffer

Columbia Virtual Internet Program (2014). Retrieved from https://www.careereducation.columbia.edu/findajob/cce-internship/vip

Conroy, R., \& Khan, R. (2009). Integrating virtual internships into online classrooms. Journal of Commercial Biotechnology, 15(2), 97-112.

Crismond, D. (2001). Learning and using science ideas when doing investigate-and-redesign tasks: A study of naive, novice, and expert designers doing constrained and scaffolded design work. Journal of Research in Science Teaching, 38(7), 791-820.

Dewey, J. (1966) Democracy and education: An introduction to the philosophy of education. New York: The Free Press.

E-Learning Market Trends \& Forecasting. (2014). Retrieved from https://www.docebo.com/landing/contactform/elearning-market-trends-and-forecast-20142016-docebo-report.pdf 
Ertmer, P.A., Stepich, D.A., York, C.S., Stickman, A., Wu, X.L., Zurek, S., \& Goktas, Y. (2008). How instructional design experts use knowledge and experience to solve ill-structured problems. Performance Improvement Quarterly, 21(1), 17-42.

Ertmer, P.A., York, C.S., \& Gedik, N. (2009). Learning from the pros: How experienced designers translate instructional design models into practice. Educational Technology, 49(1), 19-27.

Garrido-Iñigo, P., \& Rodríguez-Moreno, F. (2015). The reality of virtual worlds: Pros and cons of their application to foreign language teaching. Interactive Learning Environments, 23(4), 453470.

Garrison, D. R., Anderson, T., \& Archer, W. (2010). The first decade of the community of inquiry framework: A retrospective. The Internet and Higher Education, 13(1), 5-9.

Global Workplace Analytics. (2013). Latest telecommuting statistics. Retrieved from http://globalworkplaceanalytics.com/telecommuting-statistics

Jeske, D., \& Axtell, C. (2014). e-Internships: Prevalence, characteristics and role of student perspectives. Internet Research, 24(4), 457-473.

Jeske, D., \& Axtell, C. M. (2016). Going global in small steps: E-internships in small and mediumsized organizations. Organizational Dynamics, 45(1), 55-63.

Jonassen, D.H. (1997). Instructional design models for well-structured and iII-structured problemsolving learning outcomes. Educational Technology Research and Development, 45(1), 6594.

Kozma, R.B. (1994). Will media influence learning? Reframing the debate. Educational Technology Research and Development, 42(2), 7-19.

Kuh, G.D. (1993). In their own words: What students learn outside the classroom. American Educational Research Journal, 30(2), 277-304.

Mayernik, M.S., Thompson, C.A., Williams, V., Allard, S., Palmer, C.L., \& Tenopir, C. (2015). Enriching education with exemplars in practice: Iterative development of data curation internships. International Journal of Digital Curation, 1O(1), 123-134.

Mulki, J., Bardhi, F., Lassk, F., \& Nanavaty-Dahl, J. (2012). Set up remote workers to thrive. Occupational outlook handbook (2011). Washington, D.C.: Bureau of Labor Statistics. Available at http://www.bls.gov/ooh/

Perez, R.S., \& Emery, C.D. (1995). Designer thinking: How novices and experts think about instructional design. Performance Improvement Quarterly, 8(3), 80-95.

Reiser, R.A., \& Dempsey, J.V. (2011). Trends and issues in instructional design and technology (3 ${ }^{\text {rd }}$ ed.). Boston: Pearson. 
Rienties, B., Tempelaar, D., Giesbers, B., Segers, M., \& Gijselaers, W. (2014). A dynamic analysis of why learners develop a preference for autonomous learners in computer-mediated communication. Interactive Learning Environments, 22(5), 631-648.

Rowland, G. (1992). What do instructional designers actually do? An initial investigation of expert practice. Performance Improvement Quarterly, 5(2), 65-86.

Rowland, G. (1993). Designing and instructional design. Educational Technology Research and Development, 41(1), 79-91.

Ruth, S., \& Chaudhry, I. (2008). Telework: A productivity paradox? Internet Computing, IEEE, 12(6), 87-90.

Shea, P., \& Bidjerano, T. (2013). Understanding distinctions in learning in hybrid, and online environments: An empirical investigation of the community of inquiry framework. Interactive Learning Environments, 21(4), 355-370.

Shin, B., El Sawy, O.A., Sheng, O.R.L., \& Higa, K. (2000). Telework: Existing research and future directions. Journal of Organizational Computing and Electronic Commerce, 1O(2), 85-101.

Simon, H.A. (1977). The structure of ill-structured problems. In H.A. Simon, Models of discovery: And other topics in the methods of science (pp. 304-325). Dordrecht, Holland: D. Reidel Pub. Co.

Wang, Q. (2009). Designing a web-based constructivist learning environment. Interactive Learning Environments, 17(1), 1-13.

Weidman, J.C., Twale, D J., \& Stein, E.L. (Eds.). (2001). Socialization of graduate and professional students in higher education: A perilous passage? ASHE-ERIC Higher Education Report, 28(3). San Francisco: Jossey-Bass.

Weinert, C., Maier, C., Laumer, S., \& Weitzel, T. (2014, May). Does teleworking negatively influence IT professionals? An empirical analysis of IT personnel's telework-enabled stress. In Proceedings of the 52nd ACM Conference on Computers and People Research (pp. 139-147). ACM.

\section{Athabasca}

University

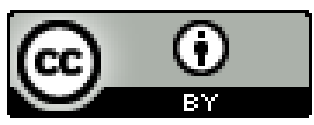

